\title{
Risiko Volksabstimmung? Zur Kluft zwischen Parteien und Wählern bei EU-Referenden in Westeuropa im Vergleich
}

\section{Kurzfassung}

Untersucht wird das Verhältnis von politischen Parteien und direktdemokratischen Verfahren exemplarisch anhand von Volksabstimmungen aus dem Kontext der Europäischen Integration. Ausgangspunkt ist die in der Integrationsforschung verbreitete These einer Kluft zwischen politischen Eliten und Bürgern beim Prozess der europäischen Einigung. Alternativ zu umfragebasierten Ansätzen wird in Anlehnung an das Konzept der Parteiendominanz direkter Demokratie die als „Partyness" konzipierte Kohärenz direktdemokratischer Ergebnisse mit repräsentativen Kräfteverhältnissen als Indikator für diese Kluft abgeleitet. Auf der Grundlage eines Original-Datensatzes von Abstimmungspositionen politischer Parteien und verschiedener Aggregatdaten wird die „Partyness“ von 31 EU- und 133 Nicht-EUAbstimmungen in Westeuropa seit 1972 ermittelt. Dieser erste umfassende Vergleich beider Abstimmungsgruppen zeigt, dass EU-Abstimmungen eine um etwa 7\% der Wahlberechtigten geringere „Partyness“ aufweisen. Anhand von Drittvariablen werden abschließend wichtige Eigenschaften der beiden Abstimmungsgruppen deutlich gemacht. So wächst im EU-Kontext die Kluft zwischen Bürgern und Parteien und die Geschlossenheit der Parteieliten entspricht viel weniger jener der Wähler als im Nicht-EU-Kontext. Das Risiko für eine geringe „Partyness“ ist bei EU-Abstimmungen größer als bei Nicht-EU-Abstimmungen. 


\section{Inhalt}

1. Einführung 236

2. Forschungsstand 237

3. Indikatorenbildung: Mit der Stimmenpotentialanalyse zur „Partyness“ direktdemokratischer Entscheidungen

4. Datengrundlage: Die „Partyness“"von 164 EU-Abstimmungen und NichtEU-Abstimmungen

5. Vergleich beider Abstimmungsgruppen anhand von Drittvariablen 248

6. Fazit

\section{Einführung}

Wesentlich zur Verbreitung und Etablierung direktdemokratischer Verfahren in den repräsentativen Systemen Europas hat der Prozess der Europäischen Integration beigetragen. Länder wie Luxemburg, die Niederlande oder Finnland haben ihre direktdemokratischen Premieren auf nationaler Ebene erst in diesem Kontext erlebt. Besondere Aufmerksamkeit haben die Abstimmungen 2005 in Frankreich und den Niederlanden erlangt, als der Entwurf des Vertrages über eine gemeinsame Europäische Verfassung (VVE) abgelehnt wurde.

Passenderweise wird in der Forschungsliteratur der Europäischen Integration ein Elite-Gesellschaft-Konflikt attestiert und diese als Elitenprojekt wahrgenommen (z. B. Immerfall 2006). Haller spricht von einer zunehmenden Kluft zwischen Eliten und Bürgern in der Haltung zur europäischen Integration: „Diese Kluft trat in aller Schärfe Anfang Mai/Ende Juni 2005 hervor, als klare Mehrheiten der Franzosen und Niederländer die Verfassung für Europa ablehnten“" (Haller 2009: 23). Die Bürger scheinen nicht so integrationswillig zu sein wie ihre Repräsentanten in den Parlamenten, und die EU-Abstimmungen befördern dies ans Tageslicht.

Von Seiten der Theorie der direkten Demokratie wurde dies aber bisher nicht umfassend bestätigt, wie die Diskussion der Forschungsliteratur im zweiten Abschnitt zeigt. Festgehalten wird dort, dass größere (aussagekräftige) Vergleiche zwischen EU-Abstimmungen und Nicht-EU-Abstimmungen aufgrund der Fokussierung auf Individualdaten fehlen. Die Rolle kollektiver Akteure kann nur begrenzt jenseits von Umfragen sichtbar gemacht werden. In jedem Fall ist die These der Kluft aber effektiv nur über den Vergleich von EU- und Nicht-EU-Abstimmungen zu überprüfen, was bislang ausgeblieben ist. Vielleicht ist ja bei Nicht-EU-Vorlagen 
Risiko Volksabstimmung?

die Kluft genauso groß. Im vorliegenden Aufsatz wird daher ein entsprechender Vergleich vorgenommen.

Dazu wird im dritten Abschnitt der Indikator für die Bemessung der Kluft zwischen Bürgern und Eliten eingeführt. Der Elitenbegriff wird auf parteipolitische Eliten verengt, was mit den verbreiteten „Party Government“-Strukturen in Westeuropa (vgl. Abromeit/Stoiber 2006) begründet werden kann. In Anlehnung an das Konzept der Parteiendominanz direkter Demokratie (Hornig 2011) wird das Ausmaß der "Partyness“ der direktdemokratischen Abstimmungsergebnisse als Indikator für die Kluft zwischen Wählern und Parteieliten herangezogen. Anhand von Aggregatdaten kann die Kohärenz eines jeden direktdemokratischen Ergebnisses mit den entsprechenden Positionen der politischen Parteien gemessen werden. Darin enthalten ist ein Perspektivwechsel weg von den Mechanismen der individuellen Abstimmungsentscheidung hin zur Kompatibilität mit den Positionen der repräsentativen Akteure. Je mehr sich die direktdemokratischen Abstimmungsergebnisse von diesen unterscheiden, desto größer die Kluft.

Im vierten Abschnitt wird für Stichproben von 31 EU- und 133 Nicht-EU-Abstimmungen der vergangenen Jahrzehnte in Westeuropa der entsprechende „Partyness"-Wert ermittelt. Dem liegt ein Originaldatensatz mit den entsprechenden Abstimmungsvorgaben aller relevanten Parteien und den entsprechenden Aggregatdaten über Wahl- und Abstimmungsergebnisse zu Grunde. Während die EU-Abstimmungen sich verhältnismäßig gleichmäßig über Westeuropa verteilen, wird die Vergleichsgruppe der Nicht-EU-Abstimmungen deutlich von schweizer und italienischen Abstimmungen dominiert, da dort die beiden intensivsten Abstimmungspraktiken zu finden ist. Im fünften Abschnitt schließlich werden die Ergebnisse der beiden Abstimmungsgruppen miteinander verglichen. Ein t-Test zeigt zunächst, dass tatsächlich die „Partyness“ der EU-Abstimmungen deutlich geringer ist als jene der Nicht-EU-Abstimmungen. Die Hinzunahme von Drittvariablen im Rahmen von multiplen Regressionsanalysen vertieft abschließend den Vergleich und untermauert die These von der zunehmenden Kluft zwischen Wählern und Parteien im EUKontext.

\section{Forschungsstand}

Die Funktionsweisen direktdemokratischer Verfahren in repräsentativen Systemen und das darin enthaltene komplexe Verhältnis von Wählern und verschiedenen politischen Eliten, werden in der Forschung zur direkten Demokratie aus verschiedenen Blickwinkeln betrachtet. Die Summe der Kriterien und Erkenntnisse gibt Aus- 
kunft über die Systemverträglichkeit direkter Demokratie (Decker 2005). Zu beachten ist dabei immer, auf welcher Erklärungsebene angesetzt wird.

Die Mikro-Perspektive fokussiert auf den einzelnen Wähler und sein Handeln in der direktdemokratischen Arena. Die Erforschung seiner Motive bei direktdemokratischen Entscheidungen steht hier im Vordergrund und kollektive Akteure kommen als Einflussgröße darauf zum Tragen. Die Frage nach einer Kluft zwischen Wählern und Parteien steckt implizit in dem Aspekt der Abstimmungsmotive der Wähler und ist also eigentlich in dieser Literatur gut aufgehoben, die analog mit der Abstimmungspraxis gewachsen ist. Insbesondere im Kontext der Abstimmungen zur Europäischen Integration ist eine Reihe von Erklärungsmodellen zum Abstimmungsverhalten der Wähler entstanden. ${ }^{1}$

Vereinfacht ausgedrückt wird zwischen zwei Erklärungsmustern unterschieden. Beim ,,issue-voting" sind die inhaltlichen Erwägungen der Wähler gegenüber dem Thema ausschlaggebend. Beim ,second-order-voting“ dominieren Aspekte der nationalen (Partei-)Politik, wie etwa der so genannte Denkzettel für die jeweilige Regierung als ein mögliches Motiv. Beiden Ansätzen geht es im Kern um die Kausalität von Abstimmungsentscheidungen und ihren Ergebnissen. Im EU-Kontext gelten als Beispiele für ,second-order-elections“ die Ablehnungen der Europäischen Verfassung 2005 in Frankreich und den Niederlanden (Schild 2005), aber auch die Ablehnung des Euros in Dänemark im Jahr 2000 (Downs 2001). Eher als Prozesse der Deliberation über die Europäische Integration gelten dagegen beispielsweise die Abstimmungen über den Vertrag von Nizza in Irland in den Jahren 2001 und 2002 (Garry/Marsh/Sinnott 2005).

Der Preis für die unzweifelhaft vorhandene Erklärungskraft der Modelle ist die geringe Übertragbarkeit der Methoden. Größtenteils wird in entsprechenden Einzelfallstudien und vergleichenden Arbeiten der Abstimmungsmotivation der Bürger anhand von Umfragedaten nachgegangen. ${ }^{2}$ Der Arbeit mit Individualdaten ist geschuldet, dass die Vergleiche selten mehr als zehn Abstimmungen berücksichtigen können. So konnte Milic zwar in seiner kognitionspsychologischen Analyse den Einfluss der Schweizer Parteien auf die inhaltliche Argumentation der entsprechenden Parteianhänger deutlich machen, aber nur bei zwei Abstimmungen (Milic 2010). Umfragen beschränken sich oft auf prominente Abstimmungen wie eben im EUKontext, so dass nur ein Ausschnitt der direktdemokratischen Praxis in Europa, nicht

1 Siehe hierzu unter anderem Trechsel/Sciarini 1998; Le Duc 2002 a und 2002 b; Bützer/Marquis 2002; Binzer Hobolt 2005, 2006 a und 2006 b; Garry/Marsh/Sinnott 2005; Wagschal 2007; Svensson 2007.

2 Vergleiche Valen 1973, 1976; Petersen 1978; Pelinka 1983; Pierce/Valen/Listhaug 1983; Siune 1993; Criddle 1993; Svensson 1996; Jahn/Storsved 1995; Bjørklund 1996; Aylott 1997, 2005; Hainsworth 2006; Qvortrup 2006. 
alle Parteien, lediglich Wähler mit Parteibindung und nur die Stimmenden berücksichtigt werden können. Eine Gewichtung der Parteien fehlt ebenfalls.

Im Erkenntnisinteresse steht daneben das Handeln von kollektiven Akteuren in der direktdemokratischen Arena, wie zum Beispiel von politischen Parteien, Parlamenten und Regierungen als Institutionen demokratischer Systeme, oder Interessengruppen wie etwa Gewerkschaften. Diese kollektiven Akteure können in ihren Bereichen auch mit dem Elitenbegriff in Verbindung gebracht werden. So haben Trechsel/Sciarini (1998) für die Schweiz gezeigt, dass der Bundesrat und die Nationalratsmehrheit als repräsentative Eliten durch direktdemokratische Verfahren nicht die Kontrolle über den politischen Prozess verlieren. Andere Untersuchungen zielten auf die Frage der Motive der Auslösung von Abstimmungen durch kollektive Akteure ab (Morel 2007; Dür/Matteo 2011). Der Hintergrund ist zumeist implizit die „Herausforderung“ durch direkte Demokratie (Budge 1996; De Vreese 2006).

Die Summe der verschiedenen Erklärungsmodelle und Falluntersuchungen zeigt dabei in der Sache, dass Parteien als kollektive Akteure unter bestimmten Umständen einen relevanten bis erheblichen Einfluss auf das Abstimmungsverhalten der Wähler ausüben können. Umso erstaunlicher ist, dass sich Höglinger zufolge in der Forschung eine „rather mystifying sympathy“ für die These der Schwächung von Parteien durch ausgebaute direkte Demokratie hält (Höglinger 2008: 209). Vielleicht liegt es daran, dass die Antwort auf die Frage „Folgt das Volk?“ (Wagschal 2007: 323) größtenteils vom Thema und den konkreten Abstimmungsumständen abhängig ist, so dass der Einfluss von Parteien zu inkonstant und nicht kontrolliert abrufbar ist. Neben dem Zeitpunkt der Entscheidung oder der „political awareness“ wird der Parteieinfluss auch anhand von weiteren Hilfsindikatoren wie Aggregatdaten oder makrosoziologischen Ansätzen sichtbar gemacht (Bützer/Marquis 2002). "The question is, precisely, under what circumstances is a political issue not a 'party issue', so that the voters will act according to their own political attitudes and disregard the cues from their social class, their party or their reference group" (Svensson 2007: 164).

Angesichts der verbreiteten „Party Government“-Strukturen bzw. Formen von Parteienstaatlichkeit in Westeuropa (Abromeit/Stoiber 2006: 171) erscheint eine Einengung des Elitenbegriffs auf parteipolitische Eliten (in Regierung und Parlament) gerechtfertigt, was andere nicht ausschließt und die diesbezüglichen Unterschiede im westeuropäischen Vergleich nicht verdecken soll. Aber selbst im semipräsidentiellen Frankreich mehren sich wieder die Zeichen für das zunehmende Gewicht der Parteien im politischen System (Pütz 2005). Dieser Tatsache muss sich auch die Forschung zur direkten Demokratie stellen. Dementsprechend bezieht sich Kriesi (2006) in seiner Analyse der Rolle von politischen Eliten in direktdemokra- 
tischen Prozessen in der Schweiz explizit auf Parteienkoalitionen und macht ihre Geschlossenheit als einen wesentlichen Einflussfaktor auf die Abstimmungsergebnisse aus. Diese Einengung des Elitenbegriffes auf Parteien wird von Kriesi mit der Zuordnung von Interessengruppen in parteipolitische Lager begründet. Verglichen werden von Kriesi Varianten von Parteienkoalitionen und die Erfolgsquoten von direktdemokratischen Vorlagen vor dem Hintergrund unterschiedlicher Mobilisierungseffekte durch die Eliten. Damit rückt zugleich das Abstimmungsergebnis als Referenzgröße mehr in den Fokus. Wagschal (2007) hat in einem diesbezüglich noch weitergehenden Ansatz die Parteivorgaben und die Zustimmung in Prozent der Stimmen bei Abstimmungen verbunden, um die Bedeutung des Medianwählers $\mathrm{zu}$ ermitteln. Beiden Autoren geht es dabei um die Übereinstimmung von direktdemokratischem „output“ und dem ,input“ der Eliten. Die Quintessenz lautet: „It is sufficient to know the configuration of the partisan forces in order to predict the outcome of the vote" (Kriesi 2006: 618). Unterscheiden sich bei einer spezifischen Frage der ,input" der Eliten und der direktdemokratische „output“, dann handelt es sich offenkundig um eine Kluft. Dieser Ansatz wird im Folgenden aufgegriffen und durch die engere Anbindung an Aggregatdaten und eine weitere Differenzierung nach einzelnen Parteien weiterentwickelt.

\section{Indikatorenbildung: Mit der Stimmenpotentialanalyse zur „Partyness“ di- rektdemokratischer Entscheidungen}

Als Indikator für die Kluft zwischen den parteipolitischen Eliten und dem tatsächlichen Abstimmungsergebnis wird aus dem Parteiendominanzansatz direkter Demokratie (Hornig 2011) die „Partyness“ direktdemokratischer Entscheidungen herangezogen. Das vorzustellende Verfahren zielt nicht auf die Kausalität von Abstimmungsentscheidungen und auf die Motive der Wähler ab, sondern fokussiert auf die Kompatibilität von direktdemokratischen Ergebnissen im repräsentativen System. Der große Vorteil ist, dass so ein weitreichender Vergleich von EU-Abstimmungen und Nicht-EU-Abstimmungen bewerkstelligt werden kann.

In Anlehnung an das „Party Government“-Konzept (Katz 1986) reflektiert der Ansatz der Parteiendominanz direkter Demokratie (,Partyness of Direct Democracy" - PoDD) die Funktionsweise direktdemokratischer Verfahren in repräsentativen Systemen. Die Ausgangsthese ist, dass bei ausgeprägten „Party Government“Strukturen bzw. Parteienstaatlichkeit auch ein Einfluss von politischen Parteien auf die Funktionsweise direktdemokratischer Verfahren zu erwarten ist. Zur genauen Bestimmung der Dominanz der direktdemokratischen Praxis durch die Parteien werden in dem Ansatz verschiedene „Frames“ des Verhältnisses von repräsentativer 
Risiko Volksabstimmung?

Sphäre allgemein, politischen Parteien im Besonderen auf der einen Seite und direktdemokratischen Verfahren auf der anderen Seite in einem Ansatz mit drei Dimensionen verbunden. Die Kompetenz zur Auslösung einer Abstimmung und die Urheberschaft der Vorlage bilden zusammen mit den Umständen der Einleitung die Kriterien der ersten Dimension (PoDD1). Die Verbindlichkeit und Beständigkeit einer direktdemokratisch getroffenen Entscheidung in Jahren bilden die Kriterien der dritten Dimension (PoDD3).

Der Vorgang der eigentlichen direktdemokratischen Abstimmung bildet die zweite Dimension (PoDD2) in der Mitte. Die „Partyness“ einer Abstimmung bemisst sich nach der Kohärenz direktdemokratischer Entscheidungen mit den Positionen der repräsentativen Akteure. Handelt es sich um eine Bestätigung der repräsentativen Politik oder wird diese durch die Abstimmung behindert bzw. korrigiert? Trechsel/Sciarini formulieren dies so: „If the parliamentary majority had a perfect control over their respective electorates, the referendum arena would be meaningless“ (1998: 102). Ein effektiver Indikator für diese perfekte Kontrolle muss die parteipolitischen Konfigurationen mit dem Abstimmungsergebnis verbinden. Je größer die Kohärenz, desto größer auch die „Partyness“ einer Abstimmung als Produzent von verbindlichen Entscheidungen im politischen System.

In der Stimmenpotentialanalyse wird diese Kohärenz einfach und transparent berechnet. Sie basiert allein auf den (in der Regel) verfügbaren Aggregatdaten von Wahl- und Abstimmungsergebnissen sowie den Abstimmungsvorgaben politischer Parteien. Ausgangspunkt ist, dass Parteien in der Regel vor direktdemokratischen Entscheidungen ihre inhaltliche Position in Form einer Abstimmungsempfehlung oder Vorgabe (bzw. Abstimmungsparole in der Schweiz) an ihre Mitglieder und Anhänger bekannt geben. Die Verteilung der Abstimmungsvorgaben absolut betrachtet zeigt an, wie viel Zustimmung oder Ablehnung eine Vorlage bei den Parteien erfährt, wie also die generelle Tendenz im Parteiensystem ist. Je einheitlicher die Vorgaben, desto eher ist eine analoge Entscheidung der Wähler zu erwarten, wenn es stimmt, dass Parteien für das Abstimmungsverhalten bedeutend sind und die Geschlossenheit insgesamt eine Rolle spielt.

Die einfache Betrachtung der Vorgaben verdeckt aber variierende Voraussetzungen der Parteien hinsichtlich des potentiellen Erfolges ihrer Vorgaben. Dazu gehören Quantität und Qualität der gesellschaftlichen Verwurzelung, aber auch die Position im Parteiensystem. Wagschal hat gezeigt, dass der Schweizer Medianwähler besonderes Gewicht hat und Parteien in seiner Nähe erfolgreicher mit ihren Vorgaben sind (Wagschal 2007: 312). Andererseits kennzeichnet kleine Parteien vom politischen Rand oftmals eine besonders motivierte und mobilisierbare Mitgliedbzw. Anhängerschaft. Die einfache Betrachtung der Vorgaben muss also durch eine 
Gewichtung angepasst werden. Diese erfolgt durch die Verkopplung mit den Stimmengewichten, wie sie aus repräsentativen Wahlen ablesbar sind. Die Grundeinheit ist immer die Gesamtzahl der Wahlberechtigten, um auch das Verhalten der Nichtwähler zu berücksichtigen. Das elektorale Gewicht repräsentiert am ehesten Mobilisierungspotentiale und Kräfteverhältnisse innerhalb eines Parteiensystems. Parlamentswahlen sind zwar nicht automatisch Parteienwahlen und es können viele Motive in der Wahlentscheidung mitschwingen. Doch entscheidend ist, dass sich ein Wähler für eine Partei entschieden hat. Durch die Ankopplung der Vorgaben an das elektorale Gewicht der Parteien kann ein in Wählerstimmen gemessenes Kräfteverhältnis erstellt werden, das als Maßstab für ein berechenbares Abstimmungsergebnis dient.

\section{Abbildung 1: Darstellung der Stimmenpotentialanalyse}

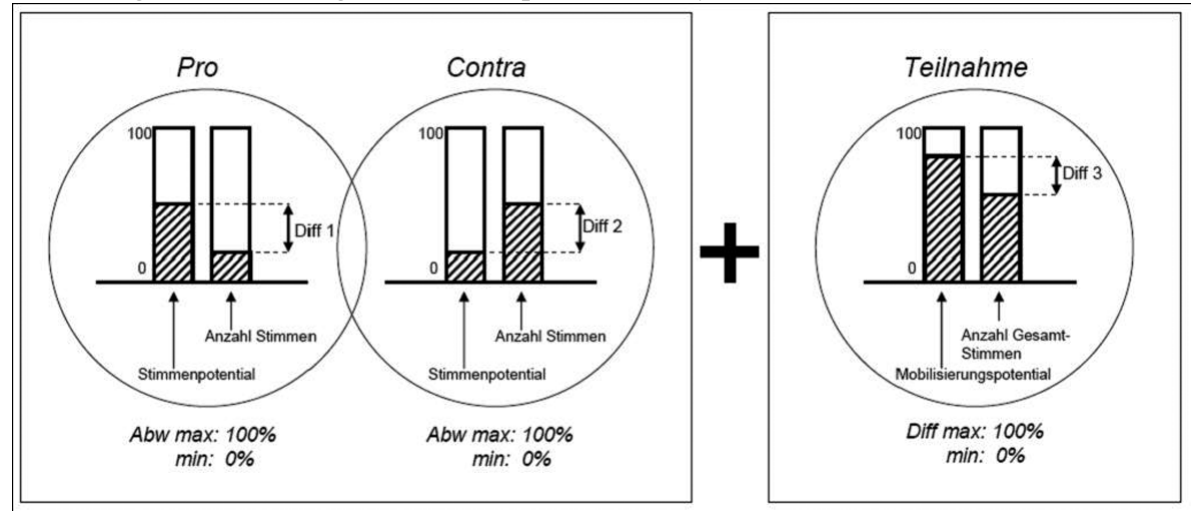

Quelle: Hornig 2011: 64.

Entsprechend ihrer Abstimmungsvorgaben werden Gruppen von Parteien gebildet und deren gemeinsamer prozentualer Stimmenanteil in Wahlberechtigten bei der letzten vorherigen Parlamentswahl addiert. Dieser Wert entspricht dem Stimmenpotential der entsprechenden Vorgabe bei der direktdemokratischen Entscheidung. Dies geschieht für die Alternativen Zustimmung und Ablehnung. Allerdings kommt es auch vor, dass Parteien entweder keine Vorgabe machen oder aber die Wähler zum Boykott der Abstimmung aufrufen. Diese freien oder Boykottvorgaben werden als dritte mögliche Variante zusammen berücksichtigt, da sie in ihrer Wirkung vergleichbar depolitisierend und demobilisierend sind. Sie sind allerdings fast ausschließlich in Italien zu finden und zielen auf das Beteiligungsquorum des dortigen abrogativen Referendums ab (Hornig 2005). Diese dritte Vorgabenart fließt nur in die Berechnung mit ein, wenn sie auch tatsächlich zum Tragen gekommen ist. An- 
Risiko Volksabstimmung?

sonsten basieren die Berechnungen allein auf den Pro- und Contra-Stimmenpotentialen.

Die einzelnen Stimmenpotentiale in Prozent der Wahlberechtigten werden mit dem tatsächlichen Ergebnis der Volksabstimmung verglichen, das heißt das ProStimmenpotential mit den tatsächlichen Pro-Stimmen in Prozent der Wahlberechtigten usw. Die Differenz zwischen beiden Werten kann theoretisch maximal 100\% der Wahlberechtigten umfassen, wenn beispielsweise keine Partei eine Pro-Vorgabe gemacht hat und das Pro-Potential damit bei null liegt, aber alle Wahlberechtigten mit Ja stimmen. Wenn dagegen genauso viele Wahlberechtigte mit Ja stimmen, wie bei der vorherigen Parlamentswahl die Pro-Parteien gewählt haben, ergibt sich keine Differenz. Bei dieser vollständigen Übereinstimmung entspräche das Ergebnis also dem, was anhand der Vorgabenstruktur zu erwarten gewesen wäre, wenn die Wähler strikt den Vorgaben der Parteien folgten. Hier spielt also auch indirekt die Abstimmungsbeteiligung mit hinein, weil die Grundlage eben alle Wahlberechtigten sind.

Die Differenzen der Vergleiche der Vorgaben mit den tatsächlichen Ergebnissen werden schließlich addiert und durch zwei bzw. drei geteilt, entsprechend der Anzahl an Differenzen (Pro, Contra und Boykott). Es ergibt sich am Ende ein durchschnittlicher Wert der Kohärenz in Prozent der Wahlberechtigten. Stimmen inhaltliche Ausrichtung der Parteien und Abstimmungsergebnis überein, dann funktioniert die direkte Demokratie im Sinne der dominierenden Parteienkonstellation. Andernfalls kann die Abweichung als ein Elite-Basis-Konflikt interpretiert werden. Je höher die „Partyness“, desto kleiner ist die Kluft.

\section{Datengrundlage: Die „Partyness“ von 164 EU-Abstimmungen und Nicht- EU-Abstimmungen}

Der herangezogene Datensatz umfasst fast alle EU-relevanten Entscheidungen aus allen westeuropäischen Mitgliedsländern. Mit acht Abstimmungen steht hierbei Irland an erster Stelle, gefolgt von Dänemark mit sechs, Frankreich mit drei und Schweden mit zwei Abstimmungen. Die anderen Fälle sind einmalige Entscheidungen in Großbritannien, den Niederlanden, Finnland, Spanien, Malta und Italien. Für die EU-Abstimmungen ist charakteristisch, dass in einer Reihe von diesen Ländern die direktdemokratische Premiere auf nationaler Ebene im Kontext der Europäischen Integration stattfand und darüber hinaus keine weiteren Abstimmungen vorliegen. Hinzu kommen Entscheidungen aus den Nicht-Mitgliedsstaaten Schweiz (drei) und Norwegen (zwei). Bei der Heranziehung der Abstimmungsvorgaben der nationalen Schweizer Parteien ist allerdings darauf hinzuweisen, dass es auf kantonaler Ebene oftmals zu abweichenden Parolen der Kantonsparteien kommt, die 
die Einheitlichkeit und Eindeutigkeit der nationalen Parteivorgabe in Frage stellen können. Die Abstimmung über die Europäische Verfassung in Luxemburg konnte aufgrund des besonderen Wahlrechts nicht berücksichtigt werden, ${ }^{3}$ ebenso die zwei Abstimmungen über den Beitritt zur Einheitlichen Europäischen Akte (EEA) in Liechtenstein. Um eine Stichprobe handelt es sich zudem, da die direktdemokratische Praxis im EU-Kontext in Osteuropa unberücksichtigt bleibt. Mit insgesamt 31 Fällen ist die Referenzgruppe der EU-Abstimmungen knapp über dem kritischen Maß für die Annahme der Normalverteilung, so dass keine zusätzlichen Verfahrensschritte in diesem Kontext notwendig sind.

Angesichts der Fülle an direktdemokratischen Entscheidungen in Europa seit 1945 erfolgt in der Kontrollgruppe der Nicht-EU-Abstimmungen eine Beschränkung auf eine Stichprobe von 133 Entscheidungen. Diese Auswahl ist nicht ganz zufällig, sondern bedingt durch die Abstimmungspraxis entsprechender Länder und die Verfügbarkeit von Daten (besonders der Parteivorgaben). Dominiert wird die Vergleichsgruppe von 59 Abstimmungen des abrogativen Referendums in Italien, die noch um die beiden italienischen Verfassungsreferenden von 2001 und 2006 über die Regionalisierung ergänzt werden. Die zweitgrößte Gruppe stellen 57 Abstimmungen aus der Schweiz, wobei hier sowohl Verfassungsinitiativen, fakultative Referenden als auch obligatorische Referenden berücksichtigt wurden. Aufgrund dieser Menge an Daten sind im Folgenden nur die Zahlen zu den EU-Abstimmungen angegeben. Der Zeitraum der Abstimmungen erstreckt sich insgesamt vom Jahr 1972 bis 2008.

Die für die Stimmenpotentialanalyse ausgewählten Parteien sind entweder Parlamentsparteien oder haben mindestens zwei Prozent der Stimmen aller Wahlberechtigten bei der jeweils vorherigen nationalen Wahl erhalten. Die Analyse der Stimmenpotentiale der EU-Abstimmungen beruht dabei durchschnittlich auf 95,4\% der abgegebenen gültigen Stimmen der jeweils vorherigen Parlamentswahl und bildet somit eine solide Basis für die Errechnung der Übereinstimmungen mit nur einer geringen Verzerrung von durchschnittlich maximal 4,6\% der Wahlberechtigten. Einzig bei den beiden irischen Abstimmungen 2001 und 2002 ist auf eine eventuelle Verzerrung hinzuweisen. Der prozentuale Anteil liegt nur bei 87,6\% bzw. 89\% der abgegebenen gültigen Stimmen, da nicht alle Parteivorgaben ermittelt werden konnten. Alle anderen Fälle liegen über $90 \%$ der abgegebenen gültigen Stimmen. Spitzenreiter ist die Abstimmung in Norwegen von 1972 mit 99,7\%, was auf die geringe

3 Das Wahlrecht sieht vor, dass jeder Wähler so viele Stimmen zur Verfügung hat, wie in seinem Wahlkreis Parlamentsmandate zu vergeben sind, was sich wiederum nach der Bevölkerungsanzahl richtet. Demnach ergeben sich für die Parteien viel mehr Stimmen als Wahlberechtigte, so dass eine Berechnung anhand der oben vorgestellten Methode nicht erfolgen kann. 
Fragmentierung des Parteiensystems während dieser Zeit zurückzuführen ist. In der Vergleichsgruppe bildet der hohe Wert von durchschnittlich 93,7\% berücksichtigter gültiger Stimmen der jeweils vorherigen Parlamentswahl ebenfalls eine ausreichend breite Grundlage für die Stichhaltigkeit der Stimmenpotentialanalyse.

Der erste Vergleich der beiden Abstimmungsgruppen macht dabei deutlich, dass bei zehn von 31 EU-Abstimmungen die dominierende inhaltliche Tendenz der Parteivorgaben mit den direktdemokratischen Entscheidungen nicht übereinstimmte $(32,2 \%)$. In neun Fällen wurden Vorlagen abgelehnt, die von den repräsentativen Akteuren unterstützt wurden. In einem Fall wurde eine Vorlage angenommen, bei der das repräsentative Kräfteverhältnis auf eine Ablehnung hinauslief. ${ }^{4}$ Diese Ablehnungsquote ist deutlich höher als bei den Nicht-EU-Abstimmungen, wo sie nur bei 11,2\% liegt (15 von 133). Die Frage der Ablehnung oder Annahme kann aber nur partiell ein Indikator für eine Kluft zwischen Parteien und Wählern sein. So können sich Fälle ergeben, in denen die dominierende Parteivorgabe nicht mit dem Abstimmungsergebnis übereinstimmt, die aber trotzdem eine überdurchschnittliche „Partyness“ aufweisen (Beispiel Norwegen) und umgekehrt.

4 Dies war der Fall bei der Abstimmung über die Einheitliche Europäische Akte in Dänemark im Jahr 1986, bei der eine Minderheitsregierung allein für die Annahme warb. 


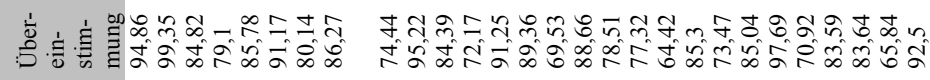

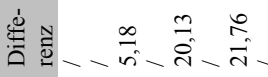
$\vec{\circ}$

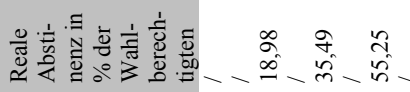

is

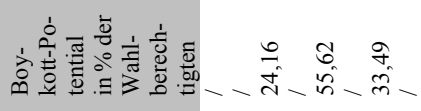

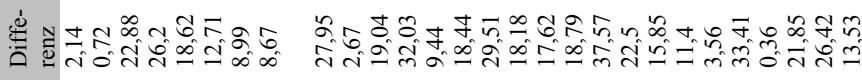

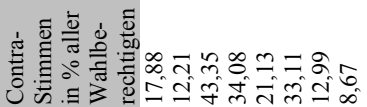

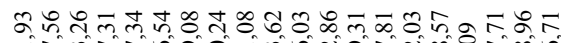

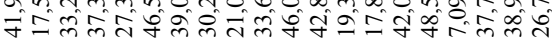

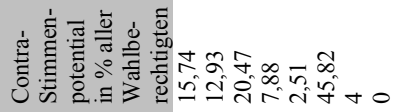

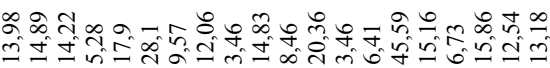

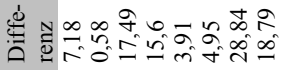

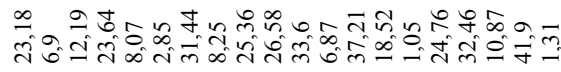

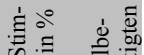
का

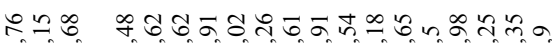

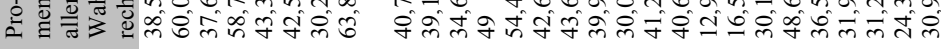

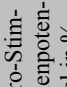

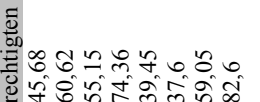

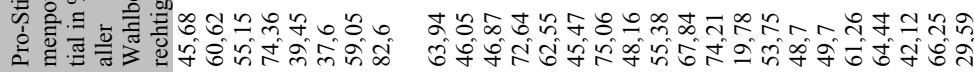
$\bar{n}$ 흘

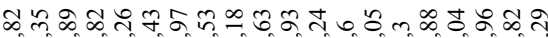

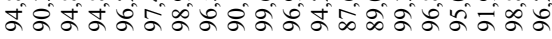

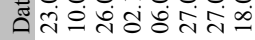

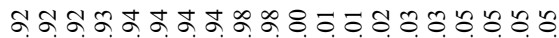

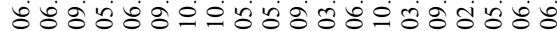

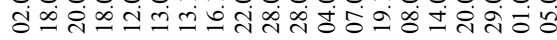

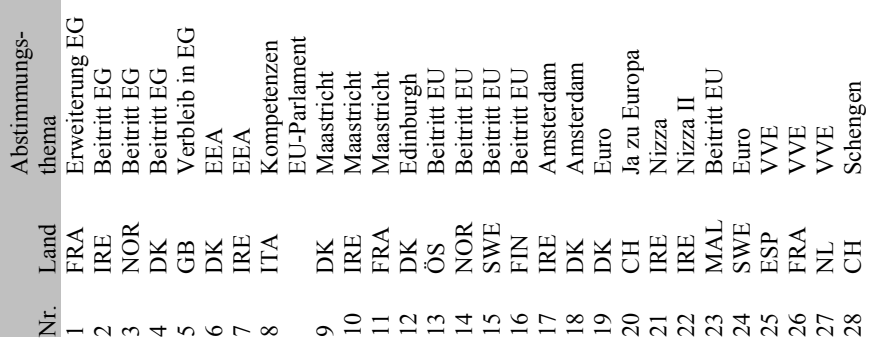




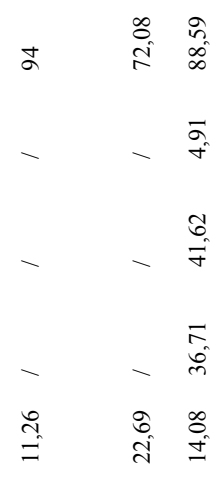

$\begin{array}{lll}\text { वे } & \text { त } & 0\end{array}$

$\stackrel{m}{\simeq} \quad \stackrel{m}{c}$

i.

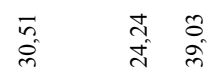

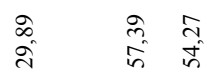

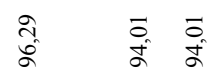

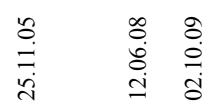

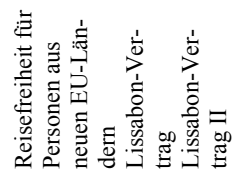

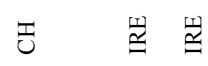

ते 이 


\section{Vergleich beider Abstimmungsgruppen anhand von Drittvariablen}

Zunächst erfolgt ein statistischer Vergleich der Mittelwerte der beiden Abstimmungsgruppen in Form eines t-Tests unabhängiger Stichproben intervallskalierter Variablen. Die zu testende Nullhypothese besagt, dass es keinen Unterschied zwischen beiden Abstimmungsgruppen gibt, während die Gegenhypothese der Ausgangsthese aus der Integrationsforschung entspricht. Die beiden Stichproben sind insofern voneinander unabhängig, als dass die Zusammensetzung der einen Gruppe keine Folgen für die Zusammensetzung der anderen Gruppe hat.

Bei den EU-Abstimmungen liegt der Mittelwert der „Partyness“ bei 83,2\% der Wahlberechtigten, bei den Nicht-EU-Abstimmungen bei 90,07\%. Die Standardabweichung beträgt in der EU-Gruppe 9,48\%, in der Kontrollgruppe nur 7,04\% der Wahlberechtigten. Die Nicht-EU-Ergebnisse sind also konstanter auf einem höheren Niveau. Das bedeutet, dass sich durchschnittlich $90 \%$ bzw. $83 \%$ der Wahlberechtigten bei den direktdemokratischen Entscheidungen so verhalten, wie es nach den repräsentativen Kräfteverhältnissen zu erwarten wäre. Diese hohen „Partyness"-Werte zeigen grundsätzlich eine sehr moderate Kluft zwischen Parteien und Abstimmenden. Ohne auf die individuellen Abstimmungsmotive eingegangen zu sein, deuten zumindest die hohen Kompatibilitätsquoten mit den Positionen aus der repräsentativen Sphäre bei den Nicht-EU-Abstimmungen darauf hin, dass Parteien in der Masse der Abstimmungen im Entscheidungsprozess der Wähler eine wichtige Rolle spielen.

Die Unterschiede in den Stichprobengruppen sind dabei nicht zufällig aufgetreten. $\mathrm{Zu}$ Beginn des t-Tests wird die Varianzgleichheit durch den Levene-Test mit einer Signifikanz von ,008 (auf einem Signifikanzniveau von $\mathrm{p}<=0,05$ ) verworfen. Auch der Prüfwert $T$ ist mit 3,79 signifikant auf einem Niveau von $p<=0,001$. Die Nullhypothese wird vor diesem Hintergrund abgelehnt, das heißt die Grundgesamtheiten der EU-Abstimmungen und der Nicht-EU-Abstimmungen haben mit der entsprechend geringen Irrtumswahrscheinlichkeit nicht denselben Mittelwert. Vielmehr liegt die Differenz zwischen den beiden Mittelwerten mit 95-prozentiger Wahrscheinlichkeit im Konfidenzintervall zwischen 3,2\% und 10,52\% der Wahlberechtigten.

Der Vergleich zwischen den beiden Abstimmungsgruppen kann durch die Einbeziehung zusätzlicher Drittvariablen in Form von verfügbaren und operationalisierbaren metrischen Daten in zwei entsprechende multiple Regressionsanalysen noch vertieft und weitere Unterschiede herausgearbeitet werden. Die Auswahl der Drittvariablen ergibt sich dabei aus den theoretischen Überlegungen im 2. Abschnitt 
bzw. aus evidenten institutionellen Unterschieden, ohne allerdings den Anspruch erheben zu können, umfassend zu sein.

So ist als erste unabhängige Variable die institutionelle Regelung der jeweiligen direktdemokratischen Verfahren zu beachten. Entsprechende politikwissenschaftliche Typologien direktdemokratischer Verfahren unterscheiden vornehmlich bezüglich der Kompetenz für die Auslösung einer Abstimmung und der Urheberschaft des Abstimmungsgegenstandes (Möckli 1994; Jung 2001; Hornig 2011). Ein Vergleich der europäischen Praxis (Kaufmann/Waters 2004) zeigt, dass die Verfahrensart auch die Nutzungsintensität bestimmt, da die wenigen offenen Verfahren vom Typ der Initiative häufiger angewandt werden als die mehrheitlich zu findenden Verfahren vom Typ eines Referendums. Dies wird relevant im Kontext der verbreiteten Annahme, dass Wähler eventuell bei nur wenigen gegebenen Abstimmungsgelegenheiten eher dazu neigen, ihren Unmut (z. B. über die Regierung) zu äußern als inhaltlich abzustimmen. Zu überprüfen wäre also, inwiefern sich diese institutionelle Ausgestaltung bei den beiden Abstimmungsgruppen unterscheidet und welchen Einfluss dies ausübt. Hinsichtlich der Operationalisierung kann festgehalten werden, dass bereits vorliegende quantitative Indizes nur die Offenheit von „bottom-up“-Verfahren messen und damit hier nur begrenzt brauchbare Werte produzieren (Stutzer/Frey 2000; Eder/Magin 2008). Ein vergleichender Bemessungsindex ist zwar auch in der ersten Dimension des Parteiendominanzansatzes (PoDD1) $\mathrm{zu}$ finden, doch bezieht sich dieser genauso allein auf die Einflussmöglichkeiten von Parteien im Ablauf der Auslösung einer Abstimmung. Möglich wäre eine dichotome Codierung aller vorliegenden Abstimmungen hinsichtlich eines ,bottomup“-oder „top-down“-Charakters in Form einer Dummy-Variable. Aufschlussreicher erscheint allerdings der Weg direkt über die Intensität der Abstimmungspraxis, da wie oben schon festgestellt die Nutzungsintensität eines einzelnen Verfahrens in der Regel die Informationen über den Verfahrenstyp mit einschließt. Dementsprechend wurde die Anzahl der Nutzung des entsprechenden Verfahrens bis zum Zeitpunkt der hier betreffenden Abstimmung als weitere unabhängige Variable berücksichtigt.

Dabei zeigt sich, dass in der Gruppe der Nicht-EU-Abstimmungen die Wähler über sehr viel mehr direktdemokratische Praxis verfügen als in der EU-Gruppe. Durchschnittlich fanden 80 Abstimmungen vor der betreffenden Nicht-EU-Abstimmung statt, wogegen es bei den EU-Abstimmungen vorher nur durchschnittlich 22,3 waren. Wie korreliert nun diese Abstimmungspraxis mit der „Partyness“? Während sich bei den Nicht-EU-Abstimmungen ein mittlerer linearer Zusammenhang von ,389 (höchst signifikant auf einem Niveau von $p<=0,001$ ) ergibt, gibt es bei den EU-Abstimmungen einen nicht signifikanten, schwachen Zusammenhang 
von ,206. Zwar ist es für die EU-Abstimmungen keine statistisch gesicherte Erkenntnis, dass mit sinkender Abstimmungshäufigkeit auch die Kluft zwischen Wählern und Parteien wächst. Dennoch ist der Trend in beiden Abstimmungsgruppen erkennbar, dass bei abnehmender Praxis auch die „Partyness“ der Abstimmungsergebnisse abnimmt. Diese Werte sind dabei teilweise mit der großen Anzahl an berücksichtigten Schweizer Abstimmungen in der Kontrollgruppe zu erklären, die zugleich eine hohe Nutzungsintensität und hohe „Partyness“-Werte aufweisen.

Der historische Zeitverlauf als zweite unabhängige Variable zeigt, inwiefern sich die „Partyness“-Werte in nahezu vierzig Jahren direktdemokratischer Praxis entwickelt haben und welche Entwicklung künftig zu erwarten ist. Im Vergleich der Korrelationen von Abstimmungsjahren und „Partyness“-Werten wird deutlich, dass sich die Vergleichsgruppen auseinander entwickeln. Bei den EU-Abstimmungen ergibt sich ein schwach negativer Zusammenhang $(-, 270)$ und bei den Nicht-EUAbstimmungen ein mittlerer positiver Zusammenhang von ,381, der zugleich auf dem Niveau von $p<=0,001$ höchst signifikant ist. Der EU-p-Wert von ,071 ist nur knapp an der Signifikanz-Grenze von 0,05 vorbei. Es bleibt zu unterstreichen, dass im Gegensatz zu den Nicht-EU-Abstimmungen die „Partyness“ der EU-Abstimmungen zunehmend sinkt. Bei weiteren Fällen ist mit einer noch wachsenden Kluft zu rechnen. In dieser zeitlichen Perspektive ist also die Ausgangsthese wiederum zu unterstreichen. Allerdings ist hier noch einmal auf die Schweizer Fälle mit ihren hohen „Partyness“-Werten in der Kontrollgruppe hinzuweisen, die alle nicht älter als aus dem Jahr 1999 sind.

Kriesi hat in der oben zitierten Arbeit auf die Bedeutung der Geschlossenheit der parteipolitischen Eliten für den Erfolg ihrer Abstimmungsvorgaben hingewiesen (2006). Zu überprüfen bleibt, wie sich dieser Faktor zu den „Partyness“-Werten der beiden Abstimmungsgruppen verhält. Bemessen wird die Geschlossenheit hier wieder auf der Grundlage von Daten aus der Stimmenpotentialanalyse. Als Indikator gilt der prozentuale Anteil des größten Stimmenpotentials in der Stimmenpotentialanalyse (in einzelnen Stimmen) an der Gesamtmenge der jeweils bei der letzten vorherigen Parlamentswahl abgegebenen gültigen Stimmen, also diesmal ohne Berücksichtigung der Nichtwähler. Der Vergleich der Mittelwerte zeigt dabei, dass die durchschnittliche Geschlossenheit der parteipolitischen Eliten bei den EU-Abstimmungen um $9 \%$ höher liegt als bei den Nicht-EU-Abstimmungen. Mit einem durchschnittlichen Gewicht der größten Stimmenpotentialgruppe von $74 \%$ weisen die parteipolitischen Eliten größtenteils einen gemeinsamen Standpunkt zur Europäischen Integration gegenüber $65 \%$ bei den Nicht-EU-Abstimmungen auf. Nur in zwei von 31 Fällen war dieser nicht zu Gunsten der EU. 
Risiko Volksabstimmung?

Tabelle 2: Statistiken des Vergleichs der zwei Abstimmungsgruppen

\begin{tabular}{|c|c|c|c|c|c|c|c|c|}
\hline & \multicolumn{4}{|c|}{ EU-Abstimmungen } & \multicolumn{4}{|c|}{ Nicht-EU-Abstimmungen } \\
\hline \multicolumn{9}{|c|}{ t-Test bei unabhängigen Stichproben } \\
\hline Mittelwert & \multicolumn{4}{|l|}{83,2} & \multicolumn{4}{|l|}{90,07} \\
\hline Standardabweichung & \multicolumn{4}{|l|}{9,48} & \multicolumn{4}{|l|}{7,04} \\
\hline \multicolumn{4}{|l|}{ (Levene-Test) F: 7,82 } & \multicolumn{5}{|c|}{ Sig. ,008 } \\
\hline \multicolumn{4}{|l|}{ T-Wert: 3,79} & \multicolumn{5}{|c|}{ Sig. (2-seitig): ,001 } \\
\hline $\begin{array}{l}\text { 95\% Konfidenzintervall } \\
\text { der Differenz }\end{array}$ & \multicolumn{4}{|l|}{ Untere: 3,20} & \multicolumn{4}{|c|}{ Obere: 10,52} \\
\hline \multicolumn{9}{|l|}{ Korrelationen } \\
\hline Unabgängige Variablen & \multicolumn{2}{|l|}{$\mathrm{r}$ (Pearson) } & \multicolumn{2}{|c|}{ Sig. (1-seitig) } & \multicolumn{2}{|l|}{$\mathrm{r}($ Pearson) } & \multicolumn{2}{|c|}{ Sig. (1-seitig) } \\
\hline $\begin{array}{l}\text { Verfahren/Abstimmungs- } \\
\text { anzahl }\end{array}$ & \multicolumn{2}{|l|}{,206 } & \multicolumn{2}{|c|}{, 134} & \multicolumn{2}{|l|}{, $389 * * *$} & \multicolumn{2}{|l|}{, 000} \\
\hline Zeitverlauf & \multicolumn{2}{|l|}{,- 270} & \multicolumn{2}{|c|}{071} & \multicolumn{2}{|l|}{, $381 * * *$} & \multicolumn{2}{|l|}{, 000} \\
\hline Geschlossenheit & \multicolumn{2}{|l|}{,$- 513 * *$} & \multicolumn{2}{|c|}{,002 } & \multicolumn{2}{|l|}{,$- 218 * *$} & \multicolumn{2}{|l|}{006} \\
\hline Wahlabstand & \multicolumn{2}{|l|}{, $334 *$} &, 03 & & 086 & &, 162 & \\
\hline Modellzusammenfassung & & & & & & & & \\
\hline $\mathrm{R}$ & ,636 & & & &, 488 & & & \\
\hline R-Quadrat &, 404 & & & &, 238 & & & \\
\hline Korrigiertes R-Quadrat &, 313 & & & &, 214 & & & \\
\hline ANOVA & F-Wert: 4,4 & & Sig & & F-Wert: 10, & $4 * * *$ & Sig.: , & \\
\hline Koeffizienten & & & & & & & & \\
\hline & $\begin{array}{l}\text { Nicht stand } \\
\text { Koeffiziente }\end{array}$ & disierte & $\begin{array}{l}\text { Stand } \\
\text { Koeffi }\end{array}$ & $\begin{array}{l}\text { ierte } \\
\text { ten }\end{array}$ & $\begin{array}{l}\text { Nicht standa } \\
\text { Koeffiziente }\end{array}$ & lisierte & $\begin{array}{l}\text { Standard } \\
\text { Koeffizi }\end{array}$ & ierte \\
\hline & $\begin{array}{l}\text { Regres- } \\
\text { sionsko- } \\
\text { effizient B }\end{array}$ & $\begin{array}{l}\text { Stan- } \\
\text { dard- } \\
\text { fehler }\end{array}$ & Beta & Sig. & $\begin{array}{l}\text { Regres- } \\
\text { sionsko- } \\
\text { effizient B }\end{array}$ & $\begin{array}{l}\text { Stan- } \\
\text { dard- } \\
\text { fehler }\end{array}$ & Beta & Sig. \\
\hline Konstante & 606,879 & 277,079 & & & $-130,588$ & 128,042 & & \\
\hline Abstimmungsanzahl &, 068 & 043 &, 287 &, 121 &, 030 & 012 &, $280^{* *}$ &, 010 \\
\hline Zeitverlauf &,- 257 &, 140 &,- 307 &, 079 &, 113 &, 064 &, 188 &, 082 \\
\hline Geschlossenheit &,- 235 &, 123 &,- 326 &, 068 &,- 110 &, 036 &,$- 237 * *$ &, 003 \\
\hline Abstand &, 176 &, 101 &, 285 &, 093 & 0,37 &, 041 &, 071 & ,364 \\
\hline $\mathrm{N}$ & 31 & & & & 133 & & & \\
\hline
\end{tabular}

${ }^{*} \mathrm{p}<=0,05 ; * *_{\mathrm{p}}<=0,01 ; * * * \mathrm{p}<=0,001$ 
Zwischen Geschlossenheit und „Partyness“ zeigt sich bei beiden Gruppen ein negativer Zusammenhang, der mit einem Wert von -,513 bei den EU-Abstimmungen bereits stark ist, bei den Nicht-EU-Abstimmungen mit -,218 aber schwach ausfällt. Zugleich sind beide Werte signifikant auf einem Niveau von $\mathrm{p}<=0,01$, so dass zufällige Ergebnisse ausgeschlossen werden können. Je geschlossener die parteipolitischen Eliten bei EU-Fragen also sind, desto mehr sinkt die „Partyness" der Ergebnisse, desto größer wird die Kluft zwischen Wählern und Parteien. Dieser Effekt ist nicht einmal halb so stark ausgeprägt wie bei Nicht-EU-Abstimmungen. Es gibt offenkundig eine Zustimmung innerhalb der Bevölkerungen, die aber ab einer bestimmten Schwelle nicht mehr zunimmt, während die Geschlossenheit der parteipolitischen Eliten darüber hinaus geht. Die Wähler sind bei EU-Abstimmungen deutlich gespaltener in ihrem Urteil als die parteipolitischen Eliten, das heißt die Geschlossenheit der parteipolitischen Eliten und die Geschlossenheit der Wähler stimmen deutlich weniger überein als bei Nicht-EU-Abstimmungen. Das heißt auch, dass in parteipolitisch umstrittenen Fällen die parteipolitisch orientierte Entscheidung bei den Wählern zunimmt, so dass höhere „Partyness“-Werte entstehen. Allerdings könnte auch bei beiden Abstimmungsgruppen ein Demobilisierungseffekt hineinspielen, da bei parteipolitisch unumstrittenen Vorlagen, die durch die große Geschlossenheit offenkundig werden, die Beteiligung an den Abstimmungen sinkt, wodurch automatisch der „Partyness“"-Wert sinkt.

Überprüft wird abschließend das Verhältnis vom Abstand zur letzten vorherigen nationalen Parlamentswahl (gemessen in Monaten) und den „Partyness“"-Werten der Abstimmungsgruppen. Sinkt mit zunehmender Distanz die „Partyness“? Im Sinne einer „Mid-term-election“ könnten Wähler geneigt sein, der Regierung mit zunehmendem Abstand zur vorherigen Wahl einen Dämpfer zu verpassen, wie es in der Diskussion der Literatur im zweiten Abschnitt als Motiv angeführt wurde. Durchschnittlich finden EU-Abstimmungen 24,8 Monate nach der letzten vorherigen Parlamentswahl statt, Nicht-EU-Abstimmungen 22,8 Monate. Die Bezeichnung „Midterm-election" scheint hier zumindest zeitlich angebracht zu sein. Bei den EU-Abstimmungen ergibt sich aber ein mittlerer Zusammenhang von ,334, der auf einem Niveau von $p<=0,05$ signifikant ist. Bei den Nicht-EU-Abstimmungen ergibt sich dagegen fast kein Zusammenhang (,086), was aber statistisch nicht abgesichert ist. Es ist also eine Besonderheit der EU-Abstimmungen, dass mit zunehmender Distanz zur vorherigen Wahl die „Partyness“ der Abstimmungsergebnisse zunimmt. Der vermutete Zusammenhang stellt sich also zumindest hier nicht ein.

Insgesamt liegt die multiple Korrelation $\mathrm{R}$ der abhängigen Variablen mit allen vier Prädiktoren bei den EU-Abstimmungen bei ,636 und bei den Nicht-EU-Abstimmungen bei ,488. Die Determinantionskoeffizienten R-Quadrat zeigen an, dass 
mit den vier unabhängigen Variablen immerhin 40\% der Varianz der EU-,,Partyness"-Werte erklärt werden kann. Bei den Nicht-EU-Abstimmungen sind es allerdings nur 24\%. Beide Modelle können dabei gegen den Zufall abgesichert werden.

Bleibt noch die Frage zu beantworten, welche der unabhängigen Variablen laut der Berechnungen am ehesten die Varianz der „Partyness“-Werte in den Abstimmungsgruppen erklären kann. Aufgrund der unterschiedlichen Einheiten der unabhängigen Variablen wird dazu auf die Beta-Werte zurückgegriffen. Hierbei ergibt sich bei den EU-Abstimmungen aber das Problem, dass alle vier Konfidenzintervalle die Null mit einschließen und daher nicht signifikant sind, auch wenn die Werte zum Teil nur geringfügig über 0,05 liegen. Zudem sind die Beta-Werte nicht sehr unterschiedlich, das heißt es sticht keine unabhängige Variable wirklich heraus. Am ehesten trägt bei den EU-Abstimmungen noch die Geschlossenheit mit einem Beta-Wert von -,326 zur Erklärung der Varianz bei, gefolgt von dem Zeitverlauf mit -,307. Die anderen beiden Prädiktoren Abstimmungsanzahl und Abstand zur letzten vorherigen Wahl weisen Anteile von ,287 bzw. ,285 auf. Bei den Nicht-EUAbstimmungen ergeben sich dagegen immerhin zwei signifikante Werte, wobei diese auch nicht sehr hoch sind. Am ehesten tragen die Abstimmungsanzahl und die Geschlossenheit zur Varianzerklärung bei den Nicht-EU-Abstimmungen bei. Die Abstimmungsanzahl ist mit einem Beta-Wert von ,280 auf dem Niveau von $p<=0,05$ signifikant, während die Geschlossenheit mit Beta -,237 auf dem Niveau von $\mathrm{p}<=0,01 \mathrm{sehr}$ signifikant ist. Der Abstand zur vorherigen repräsentativen Wahl spielt dagegen bei den Nicht-EU-Abstimmungen überhaupt keine Rolle. Der Beta-Wert von ,071 kann nicht signifikant von Null verschieden sein, da auch hier die Null im Konfidenzintervall liegt. Dies gilt auch für die unabhängige Variable Zeitverlauf mit einem Beta-Wert von ,188.

Eine vollständige Erklärung der Unterschiede zwischen den beiden Abstimmungsgruppen liegt damit nicht vor, konnte aber auch nicht unter den gegebenen Umständen produziert werden. Dazu fehlen auch weitere Variablen, auf die im theoretischen Teil hingewiesen wurde, die hier aber nicht integriert werden konnten, wie beispielsweise die Kampagnenintensität oder die Informiertheit der Bürger. Dennoch sind durch die Hinzunahme der Drittvariablen wichtige Eigenschaften der beiden Abstimmungsgruppen deutlich geworden.

\section{Fazit}

Zusammenfassend können drei wesentliche Punkte hervorgehoben werden. Erstens liegt mit der Stimmenpotentialanalyse aus dem Parteiendominanzansatz eine Methode vor, mit der sich das Verhältnis von Parteien und direktdemokratischen Ver- 
fahren erfassen und die Entsprechung eines direktdemokratischen Ergebnisses gegenüber den repräsentativen Kräfteverhältnissen genau bestimmen lässt. Dabei steht nicht die kausale Verbindung von Abstimmungsverhalten und Parteivorgaben im Vordergrund, sondern die Abweichung direktdemokratischer Entscheidungen von den repräsentativen Kräfteverhältnissen. Dementsprechend wurde auch die „Partyness" von Entscheidungen als Indikator für eine Kluft zwischen Bürgern und Parteien bei direktdemokratischen Entscheidungen herangezogen. Diese Methode ist unabhängig von Umfragen, sondern beruht auf objektiven Wahl- und Abstimmungsdaten. So konnte erstmals ein umfassender Vergleich von EU-Abstimmungen und Nicht-EU-Abstimmungen durchgeführt werden, der problemlos auf der nationalen Ebene weitergeführt werden kann.

Zweitens kann festgehalten werden, dass die herangezogenen 31 EU-Abstimmungen durchschnittlich hohe „Partyness“-Werte kennzeichnet. Im Durchschnitt stimmen die Abstimmungsergebnisse zu 83,2\% (der Wahlberechtigten) mit den repräsentativen Kräfteverhältnissen überein. In der Vergleichsgruppe der 133 NichtEU-Abstimmungen liegt der entsprechende Wert im Durchschnitt bei 90,7\% der Wahlberechtigten. Wenn von einer besonderen Kluft zwischen Parteien und Bürgern bei EU-Abstimmungen gesprochen werden kann, dann liegt diese durchschnittlich um 7\% der Wahlberechtigten höher als bei anderen Abstimmungen. Auch wenn es sich nicht um Freifahrtscheine handelt, spiegeln sich doch weitgehend die repräsentativen Kräfteverhältnisse in den Abstimmungsergebnissen wider, was zumindest auch auf eine dominante Rolle der Parteien im Abstimmungsverhalten hindeutet. Allerdings ist die Erfolgsquote der repräsentativen Akteure gemessen an der dominierenden Abstimmungsvorgabe bei den EU-Abstimmungen nur ein Drittel so hoch wie bei den Nicht-EU-Abstimmungen. Dies weist wiederum auf weiteren Forschungsbedarf bei konkreten Abstimmungen hin.

Drittens zeigt die Überprüfung von Drittvariablen wichtige Unterschiede zwischen den Vergleichsgruppen, die die These einer größeren Kluft zwischen Bürgern und Parteien im EU-Kontext bestätigen. So hat sich erstens anhand des Faktors Zeitlauf herausgestellt, dass sich das Ausmaß der „Partyness“ in beiden Vergleichsgruppen in unterschiedliche Richtungen entwickelt, auch wenn der Faktor Abstand zur letzten Parlamentswahl eine ambivalente Rolle spielt. Die EU-Abstimmungen stimmen nicht mit dem allgemeinen Trend in Westeuropa überein, dass die direktdemokratischen Ergebnisse zunehmend den repräsentativen Kräfteverhältnissen entsprechen. Das bedeutet, dass die Kluft zwischen Bürgern und parteipolitischen Eliten bei den EU-Abstimmungen sogar noch zunimmt. Zudem zeigt sich diese Kluft auch darin, dass die Wähler bei EU-Abstimmungen deutlich gespaltener in ihrem Urteil sind als die parteipolitischen Eliten, das heißt die Geschlossenheit der 
Risiko Volksabstimmung?

parteipolitischen Eliten und die Geschlossenheit der Wähler stimmen deutlich weniger überein als bei Nicht-EU-Abstimmungen. Mit zunehmender Abstimmungspraxis steigt die „Partyness“ bei EU-Abstimmungen nur halb so stark an wie bei Nicht-EU-Abstimmungen. Zusammen mit dem Zeitverlauf sind dies zwei Indikatoren für ein größeres Risiko für eine niedrigere „Partyness“ der EU-Abstimmungen gegenüber den Nicht-EU-Abstimmungen.

\section{Literatur}

Abromeit, Heidrun/Stoiber, Michael, 2006: Demokratien im Vergleich, Wiesbaden. Aylott, Nicolas, 1997: Between Europe and Unity. The Case of the Swedish Social Democrats, in: West European Politics 20 (2), 119-136.

Aylott, Nicolas, 2005, Lessons Learned, Lessons Forgotten. The Swedish Referendum on EMU in September 2003, in: Government and Opposition 40 (4), 540-564.

Binzer Hobolt, Sara, 2005: When Europe Matters. The Impact of Political Information on Voting Behaviour in EU Referendums, in: Journal of Elections, Public Opinion and Parties 15 (1), 85-109.

Binzer Hobolt, Sara, 2006 a: Direct Democracy and European Integration, in: Journal of European Public Policy 13 (1), 153-166.

Binzer Hobolt, Sara, 2006 b: How Parties affect Vote Choice in European Integration Referendums, in: Party Politics 12 (5), 623-647.

Bjørklund, Tor, 1996: The Three Nordic 1994 Referenda Concerning Membership in the EU, in: Cooperation and Conflict 31 (1), 11-36.

Budge, Ian, 1996: The new challenge of direct democracy, Cambrige/Oxford.

Bützer, Michael/Marquis, Lionel, 2002: Public opinion formation in Swiss federal referendums, in: David M. Farrell/Rüdiger Schmitt-Beck (Hrsg.), Do Political Campaigns matter? Campaign effects in elections and referendums, New York/ Abingdon, 163-182.

Chimenti, Anna, 1999: Storia dei Referendum. Dal divorzio alla riforma elettorale, Rom.

Criddle, Byron, 1993: The French Referendum on the Maastricht Treaty, in: Parliamentary Affairs 46 (2), 228-238.

De Vreese, Claes H., 2006: Political parties in dire Straits? Consequences of National Referendums for political parties, in: Party Politics 12 (5), 581-598.

Decker, Frank, 2005: Die Systemverträglichkeit der direkten Demokratie, in: Zeitschrift für Politikwissenschaft 15 (4), 1103-1148. 
Downs, William M., 2001: Denmark's referendum on the euro: The mouse that roared... again, in: West European Politics 24 (1), 222-226.

Dür, Andreas/Matteo, Gemma, 2011: To Call or Not to Call? Political Parties and Referendums on the EU's Constitutional Treaty, in: Comparative Politics (i. E.). Eder, Christina/Magin, Raphael, 2008: Direkte Demokratie, in: Markus Freitag/ Adrian Vatter (Hrsg.), Die Demokratien der deutschen Bundesländer, Opladen, 257-308.

Garry, John/Marsh, Michael/Sinnott, Richard, 2005: 'Second-oder' versus 'Issuevoting' Effects in EU Referendums, in: European Union Politics 6 (2), 201-221.

Hainsworth, Paul, 2006: France says No. The 29 May 2005 Referendum on the European Constitution, in: Parliamentary Affairs 59 (1), 98-117.

Haller, Max, 2009, Die Europäische Integration als Elitenprozess. Das Ende eines Traums?, Wiesbaden.

Höglinger, Dominic, 2008: Verschafft die direkte Demokratie den Benachteiligten mehr Gehör? Der Einfluss institutioneller Rahmenbedingungen auf die mediale Präsenz politischer Akteure, in: Swiss Political Science Review 14 (2), 207-243. Hornig, Eike-Christian, 2005: Wieder scheitert das italienische Referendum an der Blockade der Parteien, in: KAS-Auslandsinformationen 10, 22-29.

Hornig, Eike-Christian, 2011: Die Parteiendominanz direkter Demokratie in Westeuropa, Baden-Baden.

Immerfall, Stefan, 2006: Europa - politisches Einigungswerk und gesellschaftliche Entwicklung. Eine Einführung, Wiesbaden.

Jahn, Detlef/Storsved, Ann-Sofie, 1995: Legitimacy through Referendum? The nearly successful Domino-Strategy of the EU-Referendums in Austria, Finland, Sweden and Norway, in: West European Politics 18 (4), 18-37.

Jung, Sabine, 2001: Die Logik direkter Demokratie, Wiesbaden.

Katz, Richard S., 1986: Party Government. A Rationalistic Conception, in: Francis G. Castles/Rudolf Wildenmann (Hrsg.), Visions and Realities of Party Government. The Future of Party Government Vol. 1., Berlin/New York, 31-76.

Kaufmann, Bruno/Waters, Dane M., 2004: Direct democracy in Europe: a comprehensive reference guide to the initiative and referendum process in Europe, Durham.

Köppl, Stefan, 2007: Das politische System Italiens. Eine Einführung, Wiesbaden. Kriesi, Hanspeter, 2006: Role of the Political Elite in Swiss direct-democratic votes, in: Party Politics 12 (5), 599-622.

Le Duc, Lawrence, 2002 a: Referendum and elections. How do campaigns differ?, in: David M. Farrell/Rüdiger Schmitt-Beck (Hrsg.), Do Political Campaigns 
Risiko Volksabstimmung?

matter? Campaign effects in elections and referendums, New York/Abingdon, 145-162.

Le Duc, Lawrence, 2002 b: Opinion change and voting behaviour in referendums, in: European Journal of Political Research 41 (6), 711-732.

Milic, Thomas, 2010: Steuern die Parteien das Volk? Der Einfluss der Parteien auf die inhaltliche Argumentation ihrer Anhängerschaften bei Schweizer Sachabstimmungen, in: Zeitschrift für Politikwissenschaft 20 (1), 3-45.

Möckli, Silvano, 1994: Nove democrazie a confronto, in: Mario Caciagli/Pier Vincenzo Uleri (Hrsg.), Democrazie e Referendum, Rom, 49-59.

Morel, Laurence, 2007: The Rise of 'Politically Obligatory' Referendums. The 2005

French Referendum in Comparative Perspective, in: West European Politics 30

(5), 1041-1067.

Pelinka, Anton, 1983: The Nuclear Power Referendum in Austria, in: Electoral Studies 2 (3), 253-261.

Petersen, Nicolaj, 1978: Attitudes towards European Integration and the Danish Common Market Referendum, in: Scandinavian Political Studies 1 (1), 23-41.

Pettersen, Per Arnt/Jenssen, Anders Todal/Listhaug, Ola, 1996: The 1994 EU Referendum in Norway: Continuity and Change, in: Scandinavian Political Studies 19 (3), 257-280.

Pierce, Roy/Valen, Henry/Listhaug, Ola, 1983: Referendum Voting Behaviour. The Norwegian and British Referenda on Membership in the European Community, in: American Journal of Political Science 27 (1), 43-63.

Pütz, Christine, 2005: „Wenn der Teufel im Beichtstuhl sitzt...“: Präsidentschaftswahlen, Parteien und Stabilität in der V. Republik, in: Joachim Schild/Henrik Uterwedde (Hrsg.), Frankreichs V. Republik. Ein Regierungssystem im Wandel, Wiesbaden, 127-144.

Qvortrup, Matt, 2006: The Three Referendums on the European Constitution Treaty in 2005, in: The Political Quarterly 77 (1), 89-97.

Schild, Joachim, 2005: Ein Sieg der Angst - das gescheiterte französische Verfassungsreferendum, in: Integration 13 (3), 187-200.

Schneider, Gerald/Weitsman, Patricia A., 1996: The Punishment Trap. Integration Referendums as Popularity Contests, in: Comparative Political Studies 28 (4), 582-607.

Stutzer, Alois/Frey, Bruno S., 2000: Stärkere Volksrechte - Zufriedenere Bürger.

Eine mikroökonometrische Untersuchung für die Schweiz, in: Swiss Political Science Review 6 (3), 1-30.

Siune, Karen, 1993: The Danes said No to the Maastricht Treaty. The Danish EC Referendum of June 1992, in: Scandinavian Political Studies 16 (1), 93-103. 
Svennson, Palle, 2002: Five Danish referendums on the European Community and European Union: A critical assessment of the Franklin thesis, in: European Journal of Political Research 41 (6), 733-750.

Svensson, Palle, 1996: Denmark: the referendum as minority protection, in: Michael Gallagher/Pier Vincenzo Uleri (Hrsg.), The Referendum Experience in Europe, Houndmills, 33-51.

Svensson, Palle, 2007: Voting Behaviour in European Constitution Process, in: Wilfried Marxer/Zoltán Tibor Pállinger/Bruno Kaufmann/Theo Schiller (Hrsg.), Direct Democracy in Europe. Developments and Prospects, Wiesbaden, 163-173.

Trechsel, Alexander H./Sciarini, Pascal, 1998: Direct democracy in Switzerland: Do elites matter?, in: European Journal of Political Research 33 (1), 99-124.

Valen, Henry, 1973: Norway: 'no' to EEC, in: Scandinavian Political Studies 8, 214-226.

Valen, Henry, 1976: National conflict structure and foreign Politics. The Impact of the EEC Issue on perceived cleavages in Norwegian Politics, in: European Journal of Political Research 4 (1), 47-82.

Wagschal, Uwe, 2007: Diskurs oder Machtpolitik. Welche Interessen setzen sich in der Direktdemokratie am erfolgreichsten durch?, in: Markus Freitag/Uwe Wagschal (Hrsg.), Direkte Demokratie. Bestandsaufnahmen und Wirkungen im internationalen Vergleich, Münster, 303- 330.

Korrespondenzanschrift:

Dr. Eike-Christian Hornig

Technische Universität Darmstadt

Institut für Politikwissenschaft

Residenzschloss

64283 Darmstadt

E-Mail: hornig@pg.tu-darmstadt.de 